\title{
MODULES WITH DESCENDING CHAIN CONDITION
}

\author{
BY \\ EBEN MATLIS
}

Introduction. Throughout this discussion the ring $R$ will be a commutative, Noetherian ring with unit. The study of modules over such a ring has, heretofore, been largely confined to the study of finitely generated modules. The purpose of this paper is to introduce the study of modules with descending chain condition (D.C.C.), and their natural generalization-modules with maximal orders. Among the main tools in the study of these modules are the analysis of injective modules carried out in [4], and the theory of duality for complete, local rings developed there. The results of the present paper guarantee the existence of a sufficient quantity of modules with D.C.C. and provide a basis for a link between the theory of such modules and the theory of finitely generated ones. The Koszul complex, with its dual nature, plays an important role in establishing this link.

In $\S 1$ we introduce the functors $X$ and $X_{M}$. By considering these functors we are able to give characterizations of modules with maximal orders; and decompose them uniquely into direct sums, where each summand depends on only a single maximal ideal. We then prove a transition theorem which enables us to pass to rings of quotients and their completions. A key result of this section is the theorem that if $A$ is an $R$-module with D.C.C., and if $I$ is an ideal of $R$, then $I A=A$ if and only if there exists an element $r \in I$ such that $r A=A$. This is the dual of a standard result for finitely generated modules.

In $\S 2$ we introduce the concepts of cosequences and dimension, primarily for modules with D.C.C. These concepts are naturally dual to the concepts of sequences and codimension for finitely generated modules. The cohomology of the Koszul complex is exploited to provide results concerning cosequences that are dual to those obtained for sequences in [2] from the homology of the Koszul complex. Furthermore, a relationship is determined between cosequences and injective dimension that is dual to the relationship between sequences and projective dimension found in [1]. The two theories are actually equivalent, yield the same global information about the ring $R$, and either theory may be used to determine the other. In fact, the dependence of the codimension of $R$ on its maximal ideals finds its strongest expression in the above mentioned duality.

In $\$ 3$ we examine the projective dimension of modules with maximal orders. We also generalize a theorem of D. Rees [5], and obtain a direct connection between modules with maximal orders and $\nu(R)=\inf \operatorname{codim} R_{M_{\alpha}}$, where $M_{\alpha}$ ranges over all maximal ideals of $R$.

Received by the editors May 4, 1960. 
We will use the following notation throughout this paper: let $A$ be an $R$-module; then we will define $E(A)$ to be the injective envelope of $A[4]$; we will denote the projective dimension of $A$ by $\mathrm{hd}_{R} A$, and the injective dimension of $A$ by ind $A$; we will let $\operatorname{Ann}_{R} A=\{r \in R \mid r A=0\}$; and if $I$ is an ideal of $R$, then $\operatorname{Ann}_{A} I=\{x \in A \mid I x=0\}$.

1. The functors $X$ and $X_{M}$.

Definitions. Let $A$ be an $R$-module and define $X(A)=\{x \in A \mid$ every prime ideal containing $\operatorname{Ann}_{R}(x)$ is maximal $\}$. We shall call $X(A)$ the maximal component of $A$. If $A=X(A)$, we shall say that $A$ has maximal orders. Let $M$ be a maximal ideal of $R$ and define $X_{M}(A)=\left\{x \in A \mid M^{n} x=0\right.$ for some integer $n>0\}$. We shall call $X_{M}(A)$ the $M$-primary component of $A$. If $A=X_{M}(A)$, we shall say that $A$ is an $M$-primary module.

It is easily seen that both $X(A)$ and $X_{M}(A)$ are submodules of $A$. Let $M^{\prime}$ be another maximal ideal of $R$. Then we have the following: $X^{2}=X$, $X_{M}^{2}=X_{M}, X X_{M}=X_{M}=X_{M} X$, and finally $X_{M} X_{M^{\prime}}=0=X_{M^{\prime}} X_{M}$. Let $B$ be another $R$-module, and $f: A \rightarrow B$ an $R$-homomorphism. We then define $X(f): X(A) \rightarrow X(B)$ (respectively, $X_{M}(f): X_{M}(A) \rightarrow X_{M}(B)$ ) to be the restriction of $f$ to $X(A)$ (respectively, the restriction of $f$ to $X_{M}(A)$ ).

Proposition 1. (1) $X$ and $X_{M}$ are covariant, left-exact, additive functors.

(2) $X$ and $X_{M}$ commute with the taking of injective envelopes and direct limits.

(3) $X(A / X(A))=0$ and $X_{M}\left(A / X_{M}(A)\right)=0$.

Proof. The proof of the proposition is direct, and we leave its verification to the reader.

Proposition 2. Let $M$ be a maximal ideal of $R$, and let $\bar{R}_{M}$ be the completion of $R_{M}$. Then if $A$ is an $M$-primary $R$-module, we have natural isomorphisms:

$$
A \cong A \otimes_{R} R_{M} \cong A \otimes_{R} \bar{R}_{M}
$$

Proof. Let $\phi: A \rightarrow A \otimes_{R} R_{M}$ be the canonical homomorphism given by $\phi(x)$ $=x \otimes 1$, where $x \in A$. Since $A$ is $M$-primary, it is uniquely divisible by the elements of $R-M$. It follows readily that $A$ is an $R_{M}$-module, and that $\phi$ is an isomorphism.

Now $A \otimes_{R} \bar{R}_{M} \cong A \otimes_{R}\left(R_{M} \otimes_{R_{M}} \bar{R}_{M}\right) \cong\left(A \otimes_{R} R_{M}\right) \otimes_{R_{M}} \bar{R}_{M} \cong A \otimes_{R_{M}} \bar{R}_{M}$. Thus we can assume that $R$ is a local ring with maximal ideal $M$ and completion $\bar{R}$. We make $A$ into an $\bar{R}$-module in the following way. Let $\bar{r} \in \bar{R}$ and $x \in A$. There is an integer $k>0$ such that $M^{k} x=0$. Let $\left\{r_{n}\right\}$ be a Cauchy sequence in $R$ such that $r_{n} \rightarrow \bar{r}$. Then we can find an integer $N>0$ such that $r_{p}-r_{m} \in M^{k}$, whenever $p, m \geqq N$. We define $\bar{r} x=r_{N} x ;$ and it is easily checked that this makes $A$ into an $\bar{R}$-module.

We now define $f: A \rightarrow A \otimes_{R} \bar{R}$ by $f(x)=x \otimes 1$; and $g: A \otimes_{R} \bar{R} \rightarrow A$ by $g(x \otimes \bar{r})=\bar{r} x$. It is clear that $g f$ is the identity on $A$. We must show that $f g$ 
is the identity on $A \otimes_{R} \bar{R}$; i.e., we must show that $\bar{r} x \otimes 1=x \otimes \bar{r}$. Now we can find an integer $j \geqq N$ such that $\bar{r}-r_{j} \in \bar{R} M^{k}$. Then $\bar{r}=r_{j}+\sum \bar{t}_{i} m_{i}$, where $\bar{t}_{i} \in \bar{R}$ and $m_{i} \in M^{k}$. Then we have $\bar{r} x \otimes 1=r_{j} x \otimes 1=x \otimes r_{j}$; and we also have $x \otimes \bar{r}$ $=\left(x \otimes r_{j}\right)+\left(x \otimes \sum \bar{t}_{i} m_{i}\right)=\left(x \otimes r_{j}\right)+\sum\left(m_{i} x \otimes \bar{t}_{i}\right)=x \otimes r_{j}$. Thus $\bar{r} x \otimes 1=x \otimes \bar{r}_{\text {, }}$ and so $f g$ is the identity on $A \otimes_{R} \bar{R}$, and we have $A \cong A \otimes_{R} \bar{R}$.

Proposition 3. Let $A$ be an $R$-module. Then the following are equivalent:

(1) A has D.C.C.

(2) $A$ is a submodule of $E_{1} \oplus \cdots \oplus E_{n}$, where $E_{i}=E\left(R / M_{i}\right)$ with $M_{i} a$ maximal ideal of $R$.

(3) A has maximal orders and finitely generated socle.

Proof. (1) $\Rightarrow(2)$. Since $A$ has D.C.C., it is an essential extension of its socle $S$, which must be finitely generated. Thus $E(A)=E(S)$; and since $S$ is a direct sum of a finite number of simple modules, we have $E(S)$ $=E_{1} \oplus \cdots \oplus E_{n}$, where $E_{i}=E\left(R / M_{i}\right)$ with $M_{i}$ a maximal ideal of $R$.

$(2) \Rightarrow(1)$. Let $M$ be a maximal ideal of $R$ and $E=E(R / M)$. It is sufficient to prove that $E$ has D.C.C. as an $R$-module. If $B$ is any $R$-submodule of $E$, then $B=X_{M}(B)$. Thus by Proposition $2, B$ is also an $\bar{R}_{M}$-module, where $\bar{R}_{M}$ is the completion of $R_{M}$. Hence the lattice of $R$-submodules of $E$ is identical with the lattice of $\bar{R}_{M}$-submodules of $E$. Since $E$ has D.C.C. as an $\bar{R}_{M}$-module [4, Corollary 4.3], $E$ also has D.C.C. as an $R$-module.

(2) and (1) $\Rightarrow(3)$. For by (1), $A$ has a finitely generated socle; and by (2), $A$ has maximal orders.

$(3) \Rightarrow(2)$. It is sufficient to prove that $A$ is an essential extension of its socle $S$. Let $x \neq 0 \in A$, and let $M$ be a maximal ideal belonging to $A \mathrm{nn}_{R}(x)$. Then there exists an element $r \in R$ such that $r \notin A n_{R}(x)$, but $M r \subset A n_{R}(x)$. Thus $r x \neq 0$ and $r x \in S$. Hence $A$ is an essential extension of $S$.

THEOREM 1. Let $A$ be an $R$-module. Then the following are equivalent:

(1) A has maximal orders.

(2) $A$ is an essential extension of its socle.

(3) $A$ is a submodule of a direct sum of $R$-modules of the form $E\left(R / M_{\alpha}\right)$, where the $M_{\alpha}$ 's are maximal ideals of $R$. of $R$.

(4) $A=\sum \oplus X_{M_{\alpha}}(A)$, where the $M_{\alpha}^{\prime}$ 's range over all of the maximal ideals

(5) Every finitely generated submodule of $A$ has finite length.

(6) $A$ is the sum of its submodules with D.C.C.

(7) $A$ is a direct limit of $R$-modules with D.C.C.

Proof. The proofs that $(1) \Rightarrow(2)$ and $(2) \Rightarrow(3)$ are virtually the same as those found in Proposition 3. The implications $(5) \Rightarrow(6)$ and $(6) \Rightarrow(7)$ are obvious. Thus we will prove that $(3) \Rightarrow(4),(4) \Rightarrow(5)$, and $(7) \Rightarrow(1)$.

$(3) \Rightarrow(4)$. Let $B=E(A)$. It follows from [4, Theorem 3.3] that $B=\sum \oplus X_{M_{\alpha}}(B)$. It is clear that $X_{M_{\alpha}}(A)=A \cap X_{M_{\alpha}}(B)$. Now let $x \in A$; then 
$x=x_{1}+\cdots+x_{n}$, where $x_{i} \in X_{M_{\alpha_{i}}}(B)$. We will prove that each $x_{i} \in A$ by induction on $n$. The assertion is trivial for $n=1$. Hence assume that $n>1$, and that we have proved the case $n-1$. Now $\operatorname{Ann}_{R}\left(x_{1}\right)+\bigcap_{j-2}^{n} \operatorname{Ann}_{R}\left(x_{j}\right)=R$. Hence $1=m_{1}+m_{2}$, where $m_{1} \in \operatorname{Ann}_{R}\left(x_{1}\right)$ and $m_{2} \in \bigcap_{j-2}^{n} \operatorname{Ann}_{R}\left(x_{j}\right)$. Thus $x_{1}=\left(m_{1}+m_{2}\right) x_{1}=m_{2} x_{1}=m_{2} x \in A$. Therefore $x_{2}+\cdots+x_{n} \in A$. By the induction hypothesis, $x_{j} \in A$ for $j=2, \cdots, n$. Thus $x_{i} \in A \cap X_{M_{\alpha_{i}}}(B)=X_{M_{\alpha_{i}}}(A)$ for $i=1, \cdots, n$. Hence $A=\sum \oplus X_{M_{\alpha}}(A)$.

$(4) \Rightarrow(5)$. Let $C$ be a finitely generated submodule of $A$. Then the socle of $C$ is finitely generated. It is clear that $C$ has maximal orders. Therefore, by Proposition 3, $C$ has D.C.C. Thus $C$ has finite length.

$(7) \Rightarrow(1)$. By Proposition 3 every $R$-module with D.C.C. has maximal orders. Since, by Proposition 1, $X$ commutes with direct limits, it follows that $A$ has maximal orders.

Corollary. Let $A$ be an $R$-module. Then we have the following:

(1) $X(A)=\sum \oplus X_{M_{\alpha}}(A)$, where $M_{\alpha}$ ranges over all of the maximal ideals of $R$; i.e., we have the functorial equation $X=\sum \oplus X_{M_{\alpha}}$.

(2) $X(A)$ is the unique maximal essential extension in $A$ of the socle of $A$.

(3) $X(A)$ is the sum of all submodules of $A$ with D.C.C., and $A / X(A)$ has no nonzero submodules with D.C.C.

Proof. (1) This is an immediate consequence of Theorem 1.

(2) Let $S$ be the socle of $A$, and let $C$ be a submodule of $A$ which is an essential extension of $S$. Then by Theorem $1, C=X(C)$; and of course $X(C) \subset X(A)$. In particular $S$ is the socle of $X(A)$; and since $X(X(A))$ $=X(A), X(A)$ is an essential extension of $S$ by Theorem 1 .

(3) This follows directly from Theorem 1 and Propositions 1 and 3.

Definition. Let $A$ be an $R$-module with D.C.C., and let $M$ be a maximal ideal of $R$. We shall say that $M$ belongs to $A$, if $X_{M}(A) \neq 0$. It then follows from Theorem 1 that there are only a finite number of maximal ideals of $R$ that belong to $A$. If $M_{1}, \cdots, M_{n}$ are these maximal ideals, then $A=\sum_{i=1}^{n} \oplus X_{M_{i}}(A)$.

Now let $M_{1}, \cdots, M_{n}$ be a finite set of maximal ideals of $R$, and let $S$ be the complement in $R$ of $U_{i=1}^{n} M_{i}$. Then $R_{S}$ is a Zariski ring, and we shall denote its completion by $\bar{R}_{S}$. If $\bar{R}_{M_{i}}$ is the completion of $R_{M_{i}}$, then $\bar{R}_{S}$ $=\sum_{i=1}^{n} \oplus \bar{R}_{M_{i}}$. Let $E_{i}=E\left(R / M_{i}\right)$, and $E=E_{1} \oplus \cdots \oplus E_{n}$.

Proposition 4. Let $A$ be an $R$-module. Then:

(1) $X(A) \otimes_{R} R_{S} \cong \sum_{i=1}^{n} \oplus X_{M_{i}}(A) \cong X(A) \otimes_{R} \bar{R}_{S}$.

(2) $\operatorname{Hom}_{R}(X(A), E) \cong \operatorname{Hom}_{\bar{R}_{S}}\left(X(A) \otimes_{R} \bar{R}_{S}, E\right)$

$$
\cong \sum_{i=1}^{n} \oplus \operatorname{Hom}_{R_{M_{i}}}^{-}\left(X_{M_{i}}(A), E_{i}\right)
$$


(3) If $X(A)$ has D.C.C., then $\operatorname{Hom}_{R}(X(A), E)$ is a finitely generated $\bar{R}_{S^{-}}$ module.

Proof. (1) Let $M$ be a maximal ideal of $R$. Then clearly $R_{M} \otimes_{R} R_{S} \cong R_{M}$, if $M$ is one of the $M_{i}^{\prime}$ 's; and $R_{M} \otimes_{R} R_{S}=0$ otherwise. Thus by Proposition 2 and Theorem 1 we have:

$$
\begin{aligned}
X(A) \otimes_{R} R_{S} & \cong\left(\sum \oplus X_{M_{\alpha}}(A)\right) \otimes_{R} R_{S} \cong \sum \oplus\left(\left(X_{M_{\alpha}}(A) \otimes_{R} R_{M_{\alpha}}\right) \otimes_{R} R_{S}\right) \\
& \cong \sum_{i=1}^{n} \oplus\left(X_{M_{i}}(A) \otimes_{R} R_{M_{i}}\right) \cong \sum_{i=1}^{n} \oplus X_{M_{i}}(A) .
\end{aligned}
$$

Since $\bar{R}_{S}=\sum_{i=1}^{n} \oplus \bar{R}_{M_{i}}$, a similar argument shows that $X(A) \otimes_{R} \bar{R}_{S}$ $\cong \sum_{i=1}^{n} \oplus X_{M_{i}}(A)$ also.

(2) By (1), $E$ is an $\bar{R}_{S}$-module. Applying [3, Proposition 2.5.2'], we have $\operatorname{Hom}_{\bar{R}_{S}}\left(X(A) \otimes_{R} \bar{R}_{S}, E\right) \cong \operatorname{Hom}_{R}\left(X(A), \operatorname{Hom}_{\bar{R}_{S}}\left(\bar{R}_{S}, E\right) \cong \operatorname{Hom}_{R}(X(A), E)\right.$. Also by (1), we have $X(A) \otimes_{R} \bar{R}_{S} \cong \sum_{i=1}^{n} \oplus X_{M_{i}}(A)$; from which it follows easily that $\operatorname{Hom}_{\bar{R}_{S}}\left(X(A) \otimes_{R} \bar{R}_{S}, E\right) \cong \sum_{i=1}^{n} \oplus \operatorname{Hom}_{\bar{R}_{M_{i}}}\left(X_{M_{i}}(A), E_{i}\right)$.

(3) If $X(A)$ has D.C.C., then so does $X_{M_{i}}(A)$; and thus by [4, Corollary

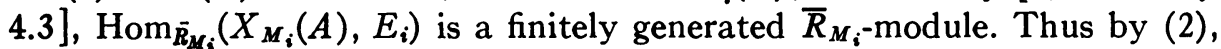
$\operatorname{Hom}_{R}(X(A), E)$ is a finitely generated $\bar{R}_{S}$-module.

Theorem 2 is the dual of the following well-known result for finitely generated modules. Let $B$ be a finitely generated $R$-module and $I$ an ideal of $R$. Then $\operatorname{Ann}_{B} I \neq 0$ if and only if every sequence $0 \rightarrow B \rightarrow^{r} B$ is not exact for every $r \in I$. Before proving Theorem 2 we shall need a lemma.

Lemma 1. Let $R$ be a Zariski ring with completion $\bar{R}$. Let $I$ be an ideal of $R$ and $P_{1}, \cdots, P_{k}$ ideals of $\bar{R}$. Then $I \subset \bigcup_{j=1}^{k} P_{j}$ if and only if $\bar{R} I \subset \bigcup_{j=1}^{k} P_{j}$.

Proof. Since $I \subset \bar{R} I$, the implication in one direction is trivial. On the other hand, assume that $I \subset \bigcup_{j=1}^{k} P_{j}$. Let $\bar{r} \in \bar{R} I$; then there exists a Cauchy sequence $\left\{r_{n}\right\}$ of elements of $I$ such that $r_{n} \rightarrow \bar{r}$. Since there are an infinite number of $r_{n}$ 's in $\bigcup_{j=1}^{k} P_{j}$, and only a finite number of $P_{j}$ 's, there exists a subsequence $\left\{r_{n}^{\prime}\right\}$ of $\left\{r_{n}\right\}$ and a $P_{m}$ such that $r_{n}^{\prime} \in P_{m}$ for all $n$. Since $r_{n}^{\prime} \rightarrow \bar{r}$, and since $P_{m}$ is closed in $\bar{R}$, it follows that $\bar{r} \in P_{m}$. Thus $\bar{R} I \subset \bigcup_{j=1}^{k} P_{j}$.

Theorem 2. Let $A$ be an R-module with D.C.C., and let I be an ideal of R. Then $I A=A$ if and only if there exists an element $r \in I$ such that $r A=A$.

Proof. If there exists $r \in I$ such that $r A=A$, then of course $I A=A$. Hence assume that $I A=A$. By Theorem 1 there exist maximal ideals $M_{1}, \cdots, M_{n}$ of $R$ such that $A=\sum_{i=1}^{n} X_{M_{i}}(A)$. Let $S$ be the complement in $R$ of $\bigcup_{i=1}^{n} M_{i}$. Then by Proposition $4, A=R_{S} A$, and so $A=\left(R_{\mathbb{S}} I\right) A$. If the theorem is true for Zariski rings, then there exists $t \in R_{S} I$ such that $t A=A$. Since $A$ is uniquely divisible by the elements of $S$, we can assume that $t \in I$, and the theorem is proved. Hence we can assume that $R$ is a Zariski ring with max- 
imal ideals $M_{1}, \cdots, M_{n}$ and completion $\bar{R}$. Let $E=\sum_{i=1}^{n} \oplus E_{i}$, where $E_{i}=E\left(R / M_{i}\right)$.

By Proposition $4, A \cong A \otimes_{R} \bar{R}$ and $\operatorname{Hom}_{R}(A, E)$ is a finitely generated $\bar{R}$-module. Let $P_{1}, \cdots, P_{k}$ be the prime ideals of $\bar{R}$ associated with $\operatorname{Hom}_{R}(A, E)$. Suppose that $r A \neq A$ for every $r \in I$. We will show that $I \subset \bigcup_{j-1}^{z} P_{j}$. For let $s \in I$. Then $A / s A \neq 0$, and so there exists a nonzero $R$ homomorphism $A / s A \rightarrow E$. Then the composition $A \rightarrow A / s A \rightarrow E$ gives a nonzero element $f \in \operatorname{Hom}_{R}(A, E)$ such that $s f=0$. Therefore, $s \in U_{j-1}^{k} P_{j}$; and hence $I \subset U_{j-1}^{k} P_{j}$. It follows from the lemma that $\bar{R} I \subset \bigcup_{j=1}^{k} P_{j}$. Therefore there is a nonzero element $g \in \operatorname{Hom}_{R}(A, E)$ such that $\bar{R} I g=0$. Thus $g(A)$ $=g(\bar{R} I A)=\bar{R} I g(A)=0$, and so $g=0$. This contradiction proves that there exists an element $r \in I$ such that $r A=A$.

Proposition 5. Let $C$ be a finitely generated $R$-module, and $A$ an $R$-module with maximal orders. Then $\operatorname{Ext}_{R}(C, A)^{n}$ and $\operatorname{Tor}^{R}(C, A)$ have maximal orders also. If $A$ has D.C.C., then so have $\operatorname{Ext}_{R}(C, A)$ and $\operatorname{Tor}_{n}^{R}(C, A)$.

Proof. Let $F$ be a finitely generated, free $R$-module such that $F \rightarrow C \rightarrow 0$ is exact. Then $0 \rightarrow \operatorname{Hom}_{R}(C, A) \rightarrow \operatorname{Hom}_{R}(F, A)$ and $F \otimes_{R} A \rightarrow C \otimes_{R} A \rightarrow 0$ are both exact. Clearly both $\operatorname{Hom}_{R}(F, A)$ and $F \otimes_{R} A$ have maximal orders; and thus both $\operatorname{Hom}_{R}(C, A)$ and $C \otimes_{R} A$ have maximal orders. Since $C$ has a projective resolution consisting of finitely generated $R$-modules, it follows readily from these remarks that both $\operatorname{Ext}_{R}(C, A)$ and $\operatorname{Tor}^{R}(C, A)$ have maximal orders.

If $A$ has D.C.C., then the same sort of argument proves the second statement of the theorem.

\section{Cosequences and dimension.}

Definition. Let $A$ be an $E$-module and $x_{1}, \cdots, x_{8}$ elements of $R$. Then we shall say that $x_{1}, \cdots, x_{\varepsilon}$ is an $A$-cosequence if:

$$
\operatorname{Ann}_{A}\left(x_{1}, \cdots, x_{i-1}\right) \stackrel{x_{i}}{\rightarrow} \operatorname{Ann}_{A}\left(x_{1}, \cdots, x_{i-1}\right) \rightarrow 0
$$

is exact for $i=1, \cdots, s$ (we use the convention that $\left(x_{1}, \cdots, x_{i-1}\right)=0$, if $i-1=0)$.

$$
\operatorname{Ann}_{A}\left(x_{1}, \cdots, x_{s}\right) \neq 0 .
$$

If $I$ is an ideal of $R$, we shall say that $x_{1}, \cdots, x_{8}$ is an $A$-cosequence in $I$ whenever $\left(x_{1}, \cdots, x_{s}\right) \subset I$. We shall call $x_{1}, \cdots, x_{s}$ a maximal $A$-cosequence in $I$ if $x_{1}, \cdots, x_{s}, y$ is not an $A$-cosequence for any $y \in I$. Since $R$ is Noetherian, it is clear that any $A$-cosequence (in $I$ ) can be extended to a maximal $A$-cosequence (in $I$ ). We note for the sake of reference that $A_{n n} I$ is naturally isomorphic to $\operatorname{Hom}_{R}(R / I, A)$.

We shall use the terms $A$-sequence and $\operatorname{codim}_{R} A$ as defined in [1] without any further comment. 
Proposition 6. Let $R$ be a complete, local ring with maximal ideal $M$ and $E=E(R / M)$. If $B$ is an $R$-module we shall define $B^{*}=\operatorname{Hom}_{R}(B, E)$. Let $A$ be an $R$-module with D.C.C. Then:

(1) $\operatorname{hd}_{R} A^{*}=\operatorname{ind}_{R} A$.

(2) If $C$ is any finitely generated $R$-module, then

$$
\mathrm{Hom}_{R}(C, A)^{*} \cong A^{*} \otimes_{R} C .
$$

(3) The elements $x_{1}, \cdots, x_{0} \in R$ are an $A$-cosequence if and only if they are an $A^{*}$-sequence.

\section{Proof.}

(1) By Proposition $4, A^{*}$ is a finitely generated $R$-module. Thus we can find a free resolution of $A^{*}$ :

$$
\cdots \rightarrow F_{n} \rightarrow \cdots \rightarrow F_{1} \rightarrow F_{0} \rightarrow A^{*} \rightarrow 0,
$$

where each $F_{i}$ is a finitely generated free $R$-module. Then $F_{i}^{*}$ is an injective $R$-module. Since $A^{* *} \cong A$ by $[4$, Corollary 4.3$]$, we obtain an injective resolution of $A$ :

$$
0 \rightarrow A \rightarrow F_{0}^{*} \rightarrow F_{1}^{*} \rightarrow \cdots \rightarrow F_{n}^{*} \rightarrow \cdots
$$

It follows that ind $A \leqq \mathrm{hd}_{R} A^{*}$.

On the other hand, by Proposition 3, we can find an injective resolution of $A$ :

$$
0 \rightarrow A \rightarrow B_{0} \rightarrow B_{1} \rightarrow \cdots \rightarrow B_{n} \rightarrow \cdots,
$$

where each $B_{i}$ is a direct sum of a finite number of copies of $E$. Then by [4, Theorem 4.2], $B_{i}^{*}$ is a free $R$-module; and so we obtain a free resolution of $A^{*}$ :

$$
\cdots \rightarrow B_{n}^{*} \rightarrow \cdots \rightarrow B_{1}^{*} \rightarrow B_{0}^{*} \rightarrow A^{*} \rightarrow 0 .
$$

Therefore, $\operatorname{hd}_{R} A^{*} \leqq \operatorname{ind}_{R} A$.

(2) Since $A^{* *} \cong A$, we have $\operatorname{Hom}_{R}\left(A^{*} \otimes_{R} C, E\right) \cong \operatorname{Hom}_{R}\left(C, A^{* *}\right)$ $\cong \operatorname{Hom}_{R}(C, A)$. Therefore, $\operatorname{Hom}_{R}(C, A)^{*} \cong\left(A^{*} \otimes_{R} C\right)^{* *}$. Since $A^{*} \otimes_{R} C$ is finitely generated, we have by $[4$, Corollary 4.3$]$ that $\left(A^{*} \otimes_{R} C\right)^{* *} \cong A^{*} \otimes_{R} C$. Thus $\operatorname{Hom}_{R}(C, A)^{*} \cong A^{*} \otimes_{R} C$.

(3) Let $I_{i}=\left(x_{1}, \cdots, x_{i}\right)$. Then by (2) we have $\left(\operatorname{Ann}_{A} I_{i}\right)^{*}$ $\cong \operatorname{Hom}_{R}\left(R / I_{i}, A\right)^{*} \cong A^{*} \otimes_{R} R / I_{i} \cong A^{*} / I_{i} A^{*}$. Since $\left(\operatorname{Ann}_{A} I_{i}\right)^{* *} \cong \operatorname{Ann}_{A} I_{i}$ by $\left[4\right.$, Corollary 4.3], we also have $\operatorname{Ann}_{A} I_{i} \cong\left(A^{*} / I_{i} A^{*}\right)^{*}$. It follows that

$$
0 \rightarrow A^{*} / I_{i} A^{*} \stackrel{x_{i+1}}{\longrightarrow} A^{*} / I_{i} A^{*}
$$

is exact if and only if

$$
\operatorname{Ann}_{A} I_{i} \stackrel{x_{i+1}}{\longrightarrow} \operatorname{Ann}_{A} I_{i} \rightarrow 0
$$


is exact. We also have that $A^{*} / I_{8} A^{*} \neq 0$ if and only if $\operatorname{Ann}_{A} I_{8} \neq 0$. Hence $x_{1}, \cdots, x_{8}$ is an $A^{*}$-sequence if and only if it is an $A$-cosequence.

THEOREM 3. Let $R$ be a local ring with maximal ideal $M$ and $E=E(R / M)$. Then $x_{1}, \cdots, x_{s} \in R$ is an $R$-sequence if and only if it is an E-cosequence.

Proof. Let $\bar{R}$ be the completion of $R$. Since $\operatorname{Hom}_{R}(E, E) \cong \bar{R}$ by $[4$, Theorem 3.7], it follows from Proposition 6 that $x_{1}, \cdots, x_{8}$ is an $E$-cosequence if and only if it is an $\bar{R}$-sequence. Since $R \cap \bar{R}\left(x_{1}, \cdots, x_{i}\right)=\left(x_{1}, \cdots, x_{i}\right)$, it is easily seen that $x_{1}, \cdots, x_{s}$ is an $\bar{R}$-sequence if and only if it is an $R$-sequence.

Definition. Let $A$ be an $R$-module, and let $x_{1}, \cdots, x_{s}$ be elements of $R$. Using the notation of [2] we define $A_{x: 1}, \ldots$, to be the Koszul complex of $A$, and $H_{j}\left(A_{x: 1}, \ldots, s\right)$ to be the $j$ th homology module of this complex. We now define the cohomology modules of this complex by $H^{i}\left(A_{x: 1}, \ldots, 8\right)$ $=H_{s-i}\left(A_{x: 1}, \ldots, s\right)$.

The proof of the following proposition may be found in [2, Proposition 1.1] and [2, Proposition 1.5]. Then:

Proposition 7. Let $A$ be an $R$-module, and $x_{1}, \cdots, x_{s}$ be elements of $R$.

$$
\begin{aligned}
H^{0}\left(A_{x: 1}, \ldots, s\right) & \cong \operatorname{Ann}_{A}\left(x_{1}, \cdots, x_{s}\right) ; \\
H^{\bullet}\left(A_{x: 1}, \ldots, s\right) & \cong A /\left(x_{1}, \cdots, x_{s}\right) A ;
\end{aligned}
$$

and

$$
H^{p}\left(A_{x: 1, \cdots, 8}\right)=0 \quad \text { for } p<0 \text { and } p>s .
$$

(2) If $C$ is a complex and $x \in R$, then we have an exact sequence:

$$
\cdots \rightarrow H^{n}\left(C_{x}\right) \rightarrow H^{n}(C) \stackrel{\delta^{n}}{\rightarrow} H^{n}(C) \rightarrow H^{n+1}\left(C_{x}\right) \rightarrow \cdots,
$$

where $\delta^{n}$ is multiplication by $(-1)^{n} x$.

(3) If $0 \rightarrow A^{\prime} \rightarrow A \rightarrow A^{\prime \prime} \rightarrow 0$ is an exact sequence of $R$-modules, then we have an exact sequence:

$$
\cdots \rightarrow H^{i}\left(A_{x: 1,}^{\prime}, \ldots, 8\right) \rightarrow H^{i}\left(A_{x: 1,}, \ldots, 8\right) \rightarrow H^{i}\left(A_{x: 1, \ldots, 8}^{\prime \prime}\right) \rightarrow H^{i+1}\left(A_{x: 1, \ldots, o}^{\prime}\right) \rightarrow \cdots .
$$

(4) $\left(x_{1}, \cdots, x_{s}\right) H\left(A_{x: 1}, \cdots, s\right)=0$.

Theorems 4 and 5 are dualized versions of [2, Proposition 2.8] and [2, Theorem 1.7].

Theorem 4. Let $A$ be an R-module with D.C.C., and let $N$ be the intersection of the maximal ideals belonging to $A$. Let $x_{1}, \cdots, x_{8}$ be elements of $N$. Then the following are equivalent:

(1) $x_{1}, \cdots, x_{8}$ is an $A$-cosequence.

(2) $H^{p}\left(A_{x: 1}, \ldots, s\right)=0$ for $p \neq 0$.

(3) $H^{1}\left(A_{x: 1, \cdots, 8}\right)=0$. 
Proof. $(1) \Rightarrow(2)$. The proof will be by induction on $s$. The case $s=0$ is trivial; hence let $s=t+1$, where $t \geqq 0$. By induction we have $H^{p}\left(A_{x: 1, \cdots, t}\right)=0$ for $p \neq 0$. Then by Proposition 7 we have an exact sequence:

$$
\operatorname{Ann}_{A}\left(x_{1}, \cdots, x_{t}\right) \stackrel{x_{t+1}}{\longrightarrow} \operatorname{Ann}_{A}\left(x_{1}, \cdots, x_{t}\right) \rightarrow H^{1}\left(A_{x: 1, \cdots, t+1}\right) \rightarrow 0 .
$$

Since the first map is an epimorphism by assumption, it follows that $H^{1}\left(A_{x: 1}, \cdots, t+1\right)=0$. By Proposition 7 we have an exact sequence for $p>0$ :

$$
H^{p}\left(A_{x: 1, \cdots, t}\right) \rightarrow H^{p+1}\left(A_{x: 1}, \cdots, t+1\right) \rightarrow H^{p+1}\left(A_{x: 1}, \cdots, t\right) .
$$

Since the end modules are zero by induction, $H^{p+1}\left(A_{x: 1}, \cdots, t+1\right)=0$ also.

$(2) \Rightarrow(3)$. This is trivial.

$(3) \Rightarrow(1)$. The proof will be by induction on $s$. For $s=1$ we have the exact sequence: $H^{0}(A) \rightarrow{ }^{x} H^{0}(A) \rightarrow H^{1}\left(A_{x}\right)$. Since $H^{0}(A)=A$ and $H^{1}\left(A_{x}\right)=0$, this is actually the exact sequence: $A \rightarrow^{x} A \rightarrow 0$. Because $x \in N$, we have $\operatorname{Ann}_{A}(x) \neq 0$ : and thus $x$ is an $A$-cosequence.

Assume we have proved the case $s=t$, and let $s=t+1$. Since $H^{1}\left(A_{x ; 1}, \cdots, t+1\right)$ $=0$, and $x_{t+1} \in N$, we conclude from the exact sequence:

$$
H^{1}\left(A_{x: 1}, \cdots, t+1\right) \rightarrow H^{1}\left(A_{x: 1}, \cdots, t\right) \stackrel{x_{t+1}}{\longrightarrow} H^{1}\left(A_{x: 1, \cdots, t}\right)
$$

that $H^{1}\left(A_{x ; 1}, \cdots, t\right)=0$. Hence by induction, $x_{1}, \cdots, x_{t}$ is an $A$-cosequence. By Proposition 7 we have the exact sequence:

$$
\operatorname{Ann}_{A}\left(x_{1}, \cdots, x_{t}\right) \stackrel{x_{t+1}}{\longrightarrow} \operatorname{Ann}_{A}\left(x_{1}, \cdots, x_{\ell}\right) \rightarrow 0 .
$$

And since $\left(x_{1}, \cdots, x_{t+1}\right) \subset N$, we have $\operatorname{Ann}_{A}\left(x_{1}, \cdots, x_{t+1}\right) \neq 0$. Thus $x_{1}, \cdots, x_{t+1}$ is an $A$-cosequence.

Corollary 2 (See [2, Corollary 2.9]). With the notation of Theorem 4 let $\pi$ be any permutation of $1, \cdots$, s. Then $x_{1}, \cdots, x_{8}$ is an $A$-cosequence if and only if $x_{\pi(1)}, \cdots, x_{\pi(s)}$ is an A-cosequence.

Corollary 3 (See [2, Proposition 2.10]). Let $R$ be a local ring with maximal $M$ and $E=E(R / M)$. Let $x_{1}, \cdots, x_{n}$ be a minimal generating set of $M$. Then $R$ is regular if and only if $H^{1}\left(E_{x: 1, \cdots, n}\right)=0$.

Proof. $H^{1}\left(E_{x ; 1}, \cdots, n\right)=0$ if and only if $x_{1}, \cdots, x_{n}$ is an $E$-cosequence by Theorem 4 , if and only if $x_{1}, \cdots, x_{n}$ is an $R$-sequence by Theorem 3 , if and only if $R$ is regular.

Theorem 5. Let $A$ be an R-module with D.C.C., and let $J$ be an ideal of $R$ such that $\mathrm{Ann}_{A} J \neq 0$. Let $x_{1}, \cdots, x_{s}$ be a maximal $A$-cosequence in $J$; and let $I=\left(y_{1}, \cdots, y_{n}\right)$ be an ideal in $J$ such that $J^{k} C I+\mathrm{Ann}_{R} A$ for some integer $k>0$. Then $s+q=n$, where $q$ is the largest integer such that $H^{q}\left(A_{y: 1, \cdots, n}\right) \neq 0$. Further, if $J \subset I+\operatorname{Ann}_{R} A$, then $H^{q}\left(A_{y: 1, \cdots, n}\right) \cong \operatorname{Ann}_{A}\left(x_{1}, \cdots, x_{s}\right) / I$ $\cdot \operatorname{Ann}_{A}\left(x_{1}, \cdots, x_{s}\right)$. 
Proof. The proof will be by induction on $s$. If $s=0$, then since Ann $J \neq 0$, it must be true that for every $x \in J$ (hence for every $x \in I$ ) the sequence $A \rightarrow^{x} A \rightarrow 0$ is not exact. But this is precisely the situation covered by Theorem 2. Thus $A \neq I A$; and therefore $H^{n}\left(A_{y: 1, \cdots, n} \cong A / I A \neq 0\right.$.

Assume the theorem is true for $s=t \geqq 0$, and let $s=t+1$. Let $B=\operatorname{Ann}_{\dot{A}}\left(x_{1}\right)$; then clearly $x_{2}, \cdots, x_{2}$ is a maximal $B$-cosequence in $J$. Thus by induction the theorem is true for $B$. From the exact sequence:

$$
0 \rightarrow B \rightarrow A \stackrel{x_{1}}{\rightarrow} A \rightarrow 0
$$

we obtain by Proposition 7 the exact sequence:

$$
H^{q}\left(A_{y: 1, \cdots, n}\right) \stackrel{x_{1}}{\rightarrow} H^{q}\left(A_{y: 1, \cdots, n}\right) \rightarrow B^{q+1}\left(B_{y: 1, \cdots, n}\right) \rightarrow 0 .
$$

If $H^{q+1}\left(B_{y: 1}, \cdots, n\right)=0$, then $H^{q}\left(A_{y: 1}, \cdots, n\right)=x_{1} H^{q}\left(A_{y: 1}, \cdots, n\right)$. By Proposition 7 we have $I H^{q}\left(A_{y: 1}, \cdots, n\right)=0=\left(\operatorname{Ann}_{R} A\right) H^{q}\left(A_{y: 1}, \cdots, n\right)$, and so $J^{k} H^{q}\left(A_{y: 1, \cdots, n}\right)$ $=0$. Therefore $H^{q}\left(A_{y: 1, \cdots, n}\right)=x_{1}^{k} H^{q}\left(A_{y: 1}, \cdots, n\right)=0$, which contradicts the assumption. Hence $H^{q+1}\left(B_{y: 1, \cdots, n}\right) \neq 0$. On the other hand it follows from the exact sequence (1) and Proposition 7 that $H^{p}\left(B_{y: 1, \cdots, n}\right)=0$ for $p>q+1$. Thus by the induction hypothesis, $t+(q+1)=n$; and so $s+q=n$.

If $J \subset I+\operatorname{Ann}_{R} A$, then $x_{1} H^{q}\left(A_{y: 1, \cdots, n}\right)=0$; and so from the exact sequence (2) and induction we obtain

$$
\begin{aligned}
H^{q}\left(A_{y: 1, \cdots, n}\right) & \cong H^{q+1}\left(B_{y: 1, \cdots, n} \cong \operatorname{Ann}_{B}\left(x_{2}, \cdots, x_{s}\right) / I \cdot \operatorname{Ann}_{B}\left(x_{2}, \cdots x_{\imath}\right)\right. \\
& \cong \operatorname{Ann}_{A}\left(x_{1}, \cdots, x_{s}\right) / I \cdot \operatorname{Ann}_{A}\left(x_{1}, \cdots, x_{s}\right) .
\end{aligned}
$$

This concludes the proof of the theorem.

Definition. Let $A$ be an $R$-module with D.C.C., and $J$ an ideal of $R$. Then we define the $J$-dimension of $A$ by $\operatorname{dim}_{J} A=\sup$ of the length of the $A$ cosequences in $J$.

Corollary 4 (See [2, Corollary 1.8]). Let $A$ be an $R$-module with D.C.C., and $J$ an ideal of $R$ such that $\mathrm{Ann}_{\mathbf{A}} J \neq 0$. Then all maximal $A$-cosequences in $J$ have the same length and $\operatorname{dim}_{J} A<\infty$. Furthermore, if $I$ is an ideal contained in $J$ such that $J \subset I+\mathrm{Ann}_{R} A$, then for any two maximal $A$-cosequences in $J, x_{1}, \cdots, x_{8}$ and $x_{1}^{\prime}, \cdots, x_{s}^{\prime}$, we have:

$$
\operatorname{Ann}_{A}\left(x_{1}, \cdots, x_{8}\right) / I \cdot \operatorname{Ann}_{A}\left(x_{1}, \cdots, x_{8}\right)
$$

$$
\cong \operatorname{Ann}_{A}\left(x_{1}^{\prime}, \cdots, x_{8}^{\prime}\right) / I \cdot \operatorname{Ann}_{A}\left(x_{1}^{\prime}, \cdots, x_{8}^{\prime}\right) \text {. }
$$

Proposition 8. Let $R$ be a local ring with maximal ideal $M$, completion $\bar{R}$, and let $\bar{M}$ be the maximal ideal of $\bar{R}$. Let $A$ be an $M$-primary $R$-module. Then:

(1) $\operatorname{Ind}_{R} A=\operatorname{Ind}_{\bar{R}} A$.

(2) If $A$ has D.C.C., then $\operatorname{dim}_{M} A=\operatorname{dim}_{\bar{M}} A$.

Proof. (1) If $E=E(R / M)$, then $E$ is also the injective envelope of $\bar{R} / \bar{M}$ 
over $\bar{R}$ by [4, Theorem 3.6]. Therefore, an $M$-primary, injective $R$-module is an $\bar{M}$-primary injective $\bar{R}$-module, and vice versa. Thus an injective resolution of $A$ as an $R$-module is the same as an injective resolution of $A$ as an $\bar{R}$-module.

(2) Let $M=\left(y_{1}, \cdots, y_{n}\right)$, and $q$ the largest integer such that $H^{q}\left(A_{y: 1}, \cdots, n\right)$ $\neq 0$. Then by Theorem $5, \operatorname{dim}_{M} A=n-q$. Since $\bar{M}=\bar{R}\left(y_{1}, \cdots, y_{n}\right)$, and since $A_{y: 1, \cdots, n}$ is the same complex, whether considered over $R$ or $\bar{R}$, it follows from Theorem 5 that $\operatorname{dim}_{\bar{M}} A=n-q$ also.

TheOREM 6. Let $R$ be a local ring with maximal ideal $M$ and $E=E(R / M)$. Let $n=\operatorname{dim}_{M} E$. Let $A$ be an R-module with D.C.C., and $x_{1}, \cdots, x_{8} \in R$ an A-cosequence. Then:

(1) $\operatorname{ind}_{R} \operatorname{Ann}_{A}\left(x_{1}, \cdots, x_{s}\right)=s+\operatorname{ind}_{R} A$.

(2) If $\operatorname{ind}_{R} A<\infty$, then $s+\operatorname{ind}_{R} A \leqq n$; and equality holds if and only if $x_{1}, \cdots, x_{8}$ is a maximal $A$-cosequence.

(3) If ind $\operatorname{in}_{R} A<\infty$, then $\operatorname{dim}_{M} A+\operatorname{ind}_{R} A=n$.

(4) If $0 \rightarrow A^{\prime} \rightarrow A \rightarrow A^{\prime \prime} \rightarrow 0$ is an exact sequence of $R$-modules such that $\operatorname{dim}_{M} A^{\prime}<\operatorname{dim}_{M} A$, then $\operatorname{dim}_{M} A^{\prime \prime}=1+\operatorname{dim}_{M} A^{\prime}$.

(5) $\operatorname{ind}_{R} R / M=$ gl. $\operatorname{dim} R=\operatorname{hd}_{R} R / M$.

Proof. This theorem could be proved directly, but for the sake of brevity we will give a proof using the results of [1]. It is clear from Proposition 8 that we can assume without loss of generality that $R$ is a complete, local ring.

(1) Using Proposition 6 and [1, Proposition 1.4], we have $\operatorname{ind}_{R} \operatorname{Ann}_{A}\left(x_{1}, \cdots, x_{s}\right)$

$$
=h d_{R} A^{*} /\left(x_{1}, \cdots, x_{s}\right) A^{*}=s+\operatorname{hd}_{R} A^{*}=s+\operatorname{ind}_{R} \mathrm{~A} .
$$

(2) By [1, Theorem 3.7] and Theorem 3,s $+\operatorname{ind}_{R} A=s+\operatorname{hd}_{R} A^{*}$ $\leqq \operatorname{codim}_{M} R=\operatorname{dim}_{M} E=n$; and equality holds if and only if $x_{1}, \cdots, x_{8}$ is a maximal $A^{*}$-sequence if and only if $x_{1}, \cdots, x_{8}$ is a maximal $A$-cosequence.

(3) This is a direct result of (2).

(4) This is proved by dualizing [1, Proposition 3.6].

(5) Since $(R / M)^{*} \cong R / M$, we have by Proposition 6 that $\operatorname{ind}_{R} R / M$ $=\operatorname{hd}_{R} R / M=$ gl. $\operatorname{dim} R$, where the last equality comes from [3, Theorem 8.6.2'].

3. Codimension; and the homological dimension of $X(A)$.

Proposition 9. Let $B$ be an $R$-module. Then $\operatorname{Ext}_{B}^{t}(S, B)=0$ for all simple $R$-modules $S$ and all $i \leqq n$ if and only if $\operatorname{Ext}_{R}^{i}(X(A), B)=0$ for all $R$-modules $A$ and all $i \leqq n$.

Proof. In one direction the implication is trivial. Hence we will assume that $\operatorname{Ext}_{R}^{i}(S, B)=0$ for all simple $R$-modules $S$ and all $i \leqq n$. Let $A$ be an $R$-module with finite length. Then there exists a submodule $A^{\prime}$ of $A$ such that $A^{\prime \prime}=A / A^{\prime}$ is simple. We have an exact sequence: 


$$
\operatorname{Ext}_{R}^{i}\left(A^{\prime \prime}, B\right) \rightarrow \operatorname{Ext}_{R}^{i}(A, B) \rightarrow \operatorname{Ext}_{R}^{i}\left(A^{\prime}, B\right)
$$

By induction on the length of $A$, the end modules are zero for $i \leqq n$. Therefore, $\operatorname{Ext}_{R}^{\prime}(A, B)=0$ for all $i \leqq n$ and all $R$-modules $A$ of finite length.

Now let $A=X(A)$, and make an induction on the integer $i$. Let $i=0$. Suppose $\operatorname{Hom}_{R}(A, B) \neq 0$. Then $B$ contains a simple submodule $S$, and $\operatorname{Hom}_{R}(S, B) \neq 0$. This contradicts the assumption, and therefore $\operatorname{Hom}_{R}(A, B)$ $=0$. We now assume the proposition true for $i-1$.

By Theorem $1, A$ is a direct limit of $R$-modules of finite length. Thus there exists an $R$-module $C$ which is a direct sum of $R$-modules of finite length, and a submodule $D$ of $C$, such that $A \cong C / D$. Hence we have the exact sequence:

$$
\operatorname{Ext}_{R}^{i-1}(D, B) \rightarrow \operatorname{Ext}_{R}^{i}(A, B) \rightarrow \operatorname{Ext}_{R}^{i}(C, B) .
$$

The first module is zero by induction, and the last module is zero by the case already proved. Hence $\operatorname{Ext}_{R}^{i}(A, B)=0$ for $i \leqq n$.

Proposition 10. Let $M$ be a maximal ideal of $R, A$ an $M$-primary $R$-module, and $B$ an $R$-module. Let $\bar{R}_{M}$ be the completion of $R_{M}$. Then:

(1) $\operatorname{Ext}_{R}^{n}(A, B) \cong \operatorname{Ext}_{R_{M}}^{n}\left(A, B \otimes_{R} R_{M}\right) \cong \operatorname{Ext}_{\underline{R}_{M}}^{n}\left(A, B \otimes_{R} \bar{R}_{M}\right)$;

(2) $\operatorname{Tor}_{n}^{R}(A, B) \cong \operatorname{Tor}_{n}^{R_{M}}\left(A, B \otimes_{R} R_{M}\right) \cong \operatorname{Tor}_{n}^{\bar{R}_{M}}\left(A, B \otimes_{R} \bar{R}_{M}\right)$; for every integer $n$.

Proof. Let $C$ be an $M$-primary $R$-module of finite length. Then $\operatorname{Ext}_{R}^{n}(C, B)$ is an $M$-primary $R$-module; and hence $\operatorname{Ext}_{R}^{n}(C, B) \cong \operatorname{Ext}_{R}^{n}(C, B)_{M}$ by Proposition 2. Thus by $\left[3\right.$, Chapter 6 , Exercise 11], we have $\operatorname{Ext}_{R_{M}}^{n}\left(C, B_{M}\right)$ $\cong \operatorname{Ext}_{R}^{n}(C, B)$. It is clear that the latter isomorphism extends to the case where $C$ is a direct sum of $M$-primary $R$-modules of finite length.

By Theorem $1, A$ is a direct limit of $M$-primary $R$-modules of finite length. Hence there exists an $R$-module $C$ which is a direct sum of $M$-primary $R$-modules of finite length, and a submodule $D$ of $C$, such that $A \cong C / D$. Thus we have a commutative diagram:

$$
\begin{aligned}
& \operatorname{Ext}_{R}^{n-1}(C, B) \longrightarrow \operatorname{Ext}_{R}^{n-1}(D, B) \longrightarrow \operatorname{Ext}_{R}^{n}(A, B) \longrightarrow \operatorname{Ext}_{R}^{n}(C, B) \longrightarrow \operatorname{Ext}_{R}^{n}(D, B) \\
& f_{1} \downarrow \quad f_{2} \downarrow \quad f_{3} \downarrow \quad f_{4} \downarrow \quad f_{5} \downarrow \\
& \operatorname{Ext}_{R_{M}}^{n-1}\left(C, B_{M}\right) \rightarrow \operatorname{Ext}_{R_{M}}^{n-1}\left(D, B_{M}\right) \rightarrow \operatorname{Ext}_{R_{M}}^{n}\left(A, B_{M}\right) \rightarrow \operatorname{Ext}_{R_{M}}^{n}\left(C, B_{M}\right) \rightarrow \operatorname{Ext}_{R_{M}}^{n}\left(D, B_{M}\right) .
\end{aligned}
$$

The rows of this diagram are exact; and $f_{1}, f_{4}$ are isomorphisms by the previous remarks. If we define $\mathrm{Ext}^{k}=0$ for $k<0$, we can begin our induction with $k=-1$, and thus we can make the induction assumption that $f_{2}$ is also an isomorphism. It follows from the "5-lemma" that $f_{3}$ is a monomorphism. The generality of this remark shows that $f_{b}$ is also a monomorphism. Then another application of the "5-lemma" shows that $f_{3}$ is an epimorphism. Thus 
we have proved that $\operatorname{Ext}_{R}^{n}(A, B) \cong \operatorname{Ext}_{R_{\mathcal{X}}}^{n}\left(A, B \otimes_{R} R_{M}\right)$ for every integer $n$. A repetition of the above argument proves that $\operatorname{Ext}_{R}^{n}(A, B) \cong \operatorname{Ext}_{\bar{R}_{M}}^{n}\left(A, B \otimes_{R} \bar{R}_{M}\right)$; and a similar argument establishes the validity of the last statement of the proposition.

Corollary 5. Let $A, B$ be arbitrary R-modules. Then:

$$
\begin{aligned}
\operatorname{Ext}_{R}^{n}(X(A), B) & \cong \prod \operatorname{Ext}_{R_{M_{\alpha}}^{n}}^{n}\left(X_{M_{\alpha}}(A), B \otimes_{R} R_{M_{\alpha}}\right) \\
& \cong \prod \operatorname{Ext}_{\bar{R}_{M_{\alpha}}}^{n}\left(X_{M_{\alpha}}(A), B \otimes_{R} \bar{R}_{M_{\alpha}}\right) \\
\operatorname{Tor}_{n}^{R}(X(A), B) & \cong \sum \oplus \operatorname{Tor}_{\bar{M}_{\alpha_{\alpha}}}^{R}\left(X_{M_{\alpha}}(A), B \otimes_{R} R_{M_{\alpha}}\right) \\
& \cong \sum \oplus \operatorname{Tor}_{n}^{R_{\mathcal{A}_{\alpha}}}\left(X_{M_{\alpha}}(A), B \otimes_{R} \bar{R}_{M_{\alpha}}\right)
\end{aligned}
$$

for all integers $n$; and where the $M_{\alpha}$ 's range over all of the maximal ideals of $R$.

Corollary 6. Let $A$ be an arbitrary $R$-module. Then $\operatorname{hd}_{R} X(A)$ $=$ sup hd ${ }_{R_{M_{\alpha}}} X_{M_{\alpha}}(A)$, where the $M_{\alpha}$ 's range over all of the maximal ideals of $R$.

Definition. We define $\nu(R)=\inf \operatorname{codim} R_{M_{\alpha}}$, where the $M_{\alpha}$ 's range over all of the maximal ideals of $R$.

THEOREM 7. $\nu(R)>n$ if and only if $\operatorname{Ext}_{R}^{\prime}(X(A), R)=0$ for all $R$-modules $A$ and for all $i \leqq n$. And in this case $\operatorname{Ext}_{R}^{i}(X(A), F)=0$ for all free $R$-modules $F$ and for all $i \leqq n$.

Proof. If $\operatorname{Ext}_{R}^{i}(R / M, R)=0$ for all $i \leqq n$, where $M$ is a maximal ideal of $R$, then by Proposition 10, $\operatorname{Ext}_{R_{M}}^{3}\left(R / M, R_{M}\right)=0$ for all $i \leqq n$. Thus by [5, Theorem 1.3], $\operatorname{codim} R_{M}>n$. Thus if $\operatorname{Ext}_{R}^{i}(X(A), R)=0$ for all $i \leqq n$, we have $\nu(R)>n$.

Conversely, assume that $\nu(R)>n$. Let $F$ be a free $R$-module. Then a trivial modification of the proof of $[5$, Theorem 1.3] shows that

$$
\operatorname{Ext}_{R_{M_{\alpha}}}^{i}\left(R_{M_{\alpha}} / M_{\alpha} R_{M_{\alpha}}, F_{M_{\alpha}}\right)=0 \quad \text { for all } i \leqq n
$$

and for all maximal ideals $M_{\alpha}$ of $R$. Hence $\operatorname{Ext}_{R}^{i}\left(R / M_{\alpha}, F\right)=0$ for all $i \leqq n$ and for all maximal ideals $M_{\alpha}$ of $R$, by Proposition 10. Therefore, $\operatorname{Ext}_{R}^{i}(X(A), F)=0$ for all $i \leqq n$ by Proposition 9 .

COROLlary 7. Let $R$ be a local ring with maximal ideal $M$. Then $R$ is regular and $\mathrm{gl} . \operatorname{dim} R=n$ if and only if $\operatorname{hd}_{R} A=n$ for all $M$-primary $R$-modules $A$.

Proof. If $h_{R} R / M=n$, then gl. $\operatorname{dim} R=n$ and $R$ is regular by [1, Theorem 1.10]. Conversely, suppose gl. $\operatorname{dim} R=n$. Then by [1, Theorem 1.9] we have $\operatorname{codim} R=n$. Let $A$ be an $M$-primary $R$-module. Then by Theorem 7, $\operatorname{Ext}_{R}^{i}(A, F)=0$ for all $i \leqq n-1$ and for every free $R$-module $F$. But $\operatorname{Ext}_{R}^{m}(A, B)$ $=0$ for all $m \geqq n+1$ and all $R$-modules $B$. Thus hd $_{R} A=n$. 


\section{BIBLIOGRAPHY}

1. M. Auslander and D. Buchsbaum, Homological dimension in local rings, Trans. Amer. Math. Soc. vol. 85 (1957) pp. 390-405.

2. - Codimension and multiplicity, Ann. of Math. vol. 68 (1958) pp. 625-657.

3. H. Cartan and S. Eilenberg, Homological algebra, Princeton, Princeton University Press, 1956.

4. E. Matlis, Injective modules over Noetherian rings, Pacific J. Math. vol. 8 (1958) pp. 511528.

5. D. Rees, The grade of an ideal or module, Proc. Cambridge Philos. Soc. vol. 53 (1957) pp. $28-42$.

NORTHWESTERN UNIVERSITY,

Evanston, Illinois 